Charm Physics (Charm 2010)

International Journal of Modern Physics: Conference Series

Vol. 2 (2011) 107-111

(C) World Scientific Publishing Company

DOI: $10.1142 / \mathrm{S} 2010194511000638$

\title{
CKM FITS AND THE ROLE OF CHARM DECAYS
}

\author{
SÉBASTIEN DESCOTES-GENON (on behalf of the CKMfitter group) \\ Laboratoire de Physique Théorique, CNRS/Univ. Paris-Sud 11, 91405 Orsay, France \\ descotes@th.u-psud.fr
}

\begin{abstract}
I review the role of charm decays in the determination of the structure of the CKM matrix within the Standard Model, focusing on $\gamma,\left|V_{c d}\right|$ and $\left|V_{c s}\right|$. In addition, I illustrate how these decays can be used to constrain some New Physics scenario, taking as an example the Two Higgs-Doublet Model of type II.
\end{abstract}

\section{Global fit of the Cabibbo-Kobayashi-Maskawa matrix}

In the Standard Model (SM), the weak charged-current transitions mix quarks of different generations, which is encoded in the unitary Cabibbo-Kobayashi-Maskawa (CKM) matrix. In the case of three generations of quarks, the physical content of this matrix reduces to four real parameters, among which one phase, the only source of $\mathrm{CP}$ violation in the Standard Model (the lepton sector can also exhibit similar sources of CP violation once neutrino masses are provided by New Physics). One can define these four real parameters as:

$$
\lambda^{2}=\frac{\left|V_{u s}\right|^{2}}{\left|V_{u d}\right|^{2}+\left|V_{u s}\right|^{2}} \quad A^{2} \lambda^{4}=\frac{\left|V_{c b}\right|^{2}}{\left|V_{u d}\right|^{2}+\left|V_{u s}\right|^{2}} \quad \bar{\rho}+i \bar{\eta}=-\frac{V_{u d} V_{u b}^{*}}{V_{c d} V_{c b}^{*}} .
$$

and exploit the unitarity of the CKM matrix to determine its entries as an expansion in $\lambda$. A challenge for both experimentalists and theorists consists in extracting information on these parameters from data in the presence of the strong interaction that binds quarks into hadrons. The CKMfitter group pursue this goal using the Rfit frequentist approach ${ }^{1}$. The current situation of the global fit is on the left hand-side of Fig. 1. A large set of constraints is involved: many, in particular those concerning the sides of the unitarity triangle, involve moduli of CKM matrix elements multiplied by hadronic quantities $\left(V_{u b}, V_{c b}, \Delta m_{d}, \Delta m_{s}, \epsilon_{K}\right)$, whereas a few others can be obtained from measurements where hadronic inputs are determined from experiment with minimal theoretical inputs $(\alpha, \beta)$.

In this game, the $\gamma$ angle stands on a particular footing compared to the other angles, since its determination is based on the interference between the colourallowed $B^{-} \rightarrow D^{0} K^{-}$and colour-suppressed $B^{-} \rightarrow \bar{D}^{0} K^{-}$decays ${ }^{2}$. The accuracy of the method is driven by the size of the ratio $r_{B}=\left|A_{\text {suppr }}\right| /\left|A_{\text {favour }}\right| \simeq$ $\left|V_{u b} V_{c s}^{*}\right| /\left|V_{c b} V_{u s}^{*}\right| \times O\left(1 / N_{c}\right)$ typically of order $0.1-0.2$, and the different methods try to improve on this ratio by different choices of $D^{(*)}$ decay channels (GLW: $D^{(*)}$ into 

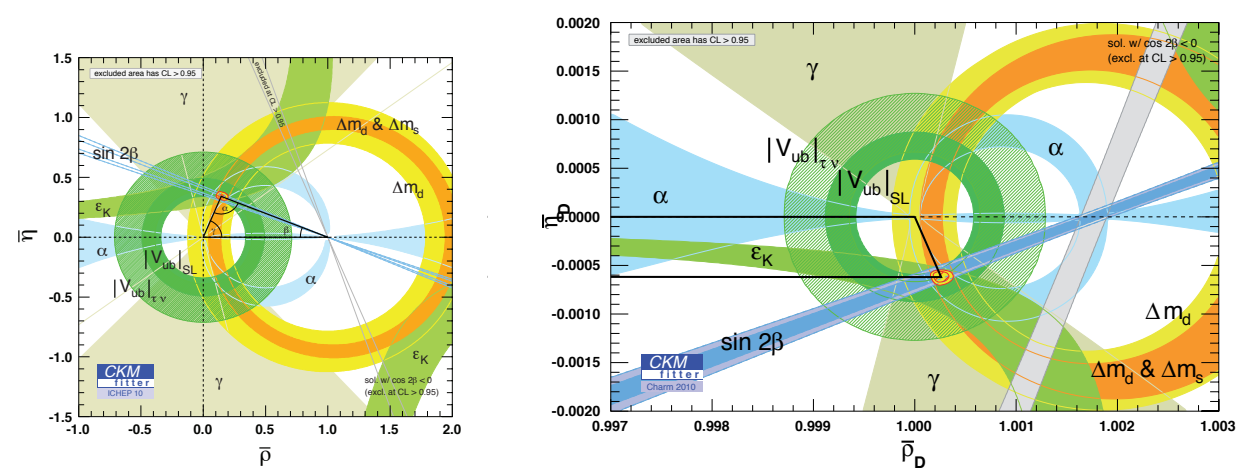

Fig. 1. Constraints on CP-violation represented in the case of the $B_{d}$-meson (left) and $D$-meson (right) unitarity triangles.

CP eigenstates, ADS: $D^{(*)}$ into doubly Cabibbo-suppressed states, GGSZ: $D^{(*)}$ into 3 -body state and Dalitz analysis). Combining the three methods yields $\gamma=\left(70_{-21}^{+14}\right)^{\circ}$ $(68 \% \mathrm{CL})$ to be compared with the prediction of the global fit $\gamma=\left(67.6_{-4.1}^{+3.6}\right)^{\circ}(68 \%$ CL). One can see in Fig. 2 that the combined value is mainly driven by the GGZ method. A central issue is the presence hadronic (nuisance) parameters ${ }^{2}$ : one has to constrain the ratio $r_{B}$ and the relative strong phase $\delta_{B}$ between the two $B$-decay amplitudes. In addition, the GLW and ADS methods involve the equivalent parameters $r_{D}$ and $\delta_{D}$ describing the weak $D$-decay amplitude. The determination of the $D$-decay parameters at BESIII ${ }^{3,4}$, and in particular the phase $\delta_{D}$, could help to reduce the uncertainty on $\gamma$ for GLW and ADS methods, as can be seen in Fig. 2.

\section{Standard Model determination of $\left|V_{c d}\right|$ and $\left|V_{c s}\right|$}

One may wonder whether other inputs from the $D$ sector can impact the determination of the CKM matrix more directly. In analogy with the $B$-meson case based on unitarity for the $(b, d)$ columns, one can use the $(u, c)$ rows:

$$
\frac{V_{u d} V_{c d}^{*}}{V_{u s} V_{c s}^{*}}+1+\frac{V_{u b} V_{c b}^{*}}{V_{u s} V_{c s}^{*}}=0 \quad O(1)+1+O\left(\lambda^{4}\right)=0
$$

to define a squashed $D$-meson unitarity triangle with an apex defined as $\bar{\rho}_{D}+i \bar{\eta}_{D}=$ $-\left(V_{u d} V_{c d}^{*}\right) /\left(V_{u s} V_{c s}^{*}\right)$. The corresponding constraints from the global fit are represented on the right hand-side of Fig. 1. $C P$-violating $D$-decay observables would clearly be required at a very high level of accuracy (both theoretically and experimentally) to compete with the constraints from the $B$-meson sector. As far as $C P$ conserving observables are concerned, charm mixing provides is difficult to exploit to determine CKM matrix parameters due to long-distance effects that are difficult to understand theoretically ${ }^{5}$. Leptonic and semileptonic $D$ decays seem much more promising $^{6}$, leading to the moduli $\left|V_{c d}\right|$ and $\left|V_{c s}\right|$ if the relevant hadronic matrix 

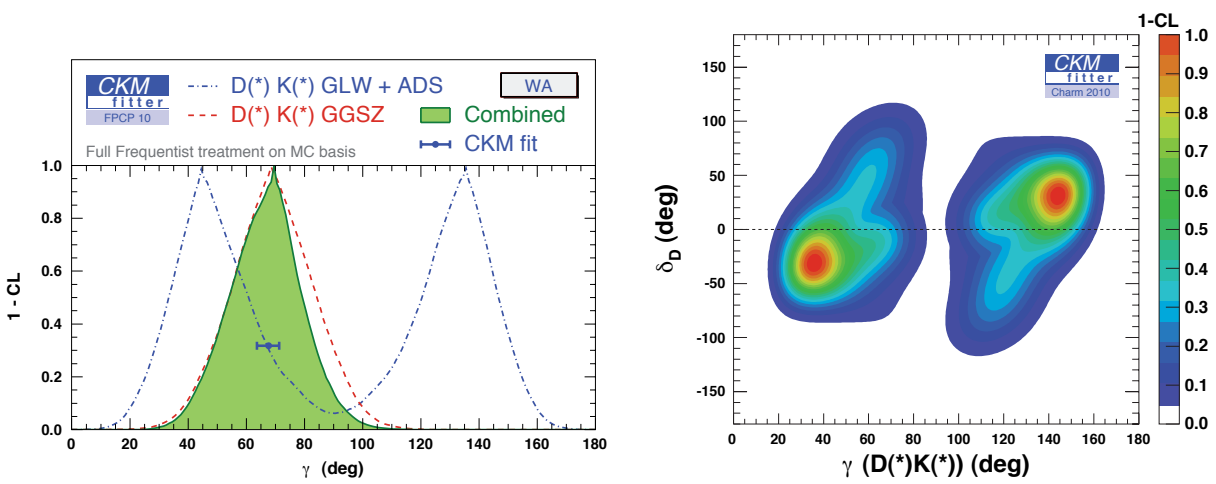

Fig. 2. On the left, current constraints on $\gamma$ using GLW and ADS methods (blue), GGSZ method (red) and a combination of the methods (green). On the right, current constraints on $\gamma$ using GLW and ADS methods as a function of the phase $\delta_{D}$, using $D K, D K^{*}$ and $D^{*} K$ decays (right).

elements are known (form factors, decay constants). We determine the latter by averaging lattice computations performed with three dynamical flavours, distinguishing between statistical and systematic uncertainties ${ }^{7}$. We obtain for the decay constants $f_{D_{s}}=251.2 \pm 1.2 \pm 4.5 \mathrm{GeV}, f_{D_{s}} / f_{D}=1.186 \pm 0.005 \pm 0.010$, and for the semileptonic form factors $F_{+}^{\pi}(0)=0.64 \pm 0.03 \pm 0.15, F_{+}^{K}(0)=0.747 \pm 0.011 \pm 0.034$.

The left hand-side of Fig. 3 shows the resulting values of $\left|V_{c d}\right|$ and $\left|V_{c s}\right|$ from these direct measurements, compared to indirect determinations relying on unitarity. Nucleon and kaon observables alone set loose constraints, since they do no contain enough information on the corrections to the equality between $\left|V_{c d}\right|$ and $\left|V_{u s}\right|$ (holding only at leading order in $\lambda$ ). The $b$-sector provides rather stringent constraints, in particular through the unitarity constraint $\left|V_{c d}\right|^{2}+\left|V_{c s}\right|^{2}=1-\left|V_{c b}\right|^{2}$ reducing the allowed area for $\left|V_{c d}\right|$ and $\left|V_{c s}\right|$ to a thin ring. We obtain for the direct (leptonic and semileptonic) measurements: $\left|V_{c d}\right|=0.225 \pm 0.008$ and $\left|V_{c s}\right|=0.955_{-0.025}^{+0.002}$, whereas the indirect constraints yield $\left|V_{c d}\right|=0.2253 \pm 0.0001,\left|V_{c s}\right|=0.9743 \pm 0.0002$.

The ongoing improvements in lattice simulations of charm hadronic quantities ${ }^{6}$ should allow interesting comparisons between the direct and indirect determinations of $\left|V_{c d}\right|$ and $\left|V_{c s}\right|$ soon. As illustrated by the right hand-side of Fig. 3, direct constraints from charm decays should be formulated through ratios of $D$ and $D_{s}$ decays, like $\Gamma(D \rightarrow \ell \nu) / \Gamma\left(D_{s} \rightarrow \ell \nu\right)$ or $\Gamma(D \rightarrow \pi e \nu) / \Gamma(D \rightarrow K e \nu)$. These ratios give access to $\left|V_{c d}\right| /\left|V_{c s}\right|$ with reduced experimental and theoretical uncertainties, and provide an orthogonal constraint to that derived from $\left|V_{c b}\right|$ and unitarity. Other modes should also be considered, in particular vector modes for semileptonic decays. For instance, $\Gamma(D \rightarrow \pi \ell \nu) / \Gamma\left(D_{s} \rightarrow \phi \ell \nu\right)$ could be very interesting, since the narrow $\phi$-meson is easier to simulate in lattice gauge theories and to identify experimentally. 

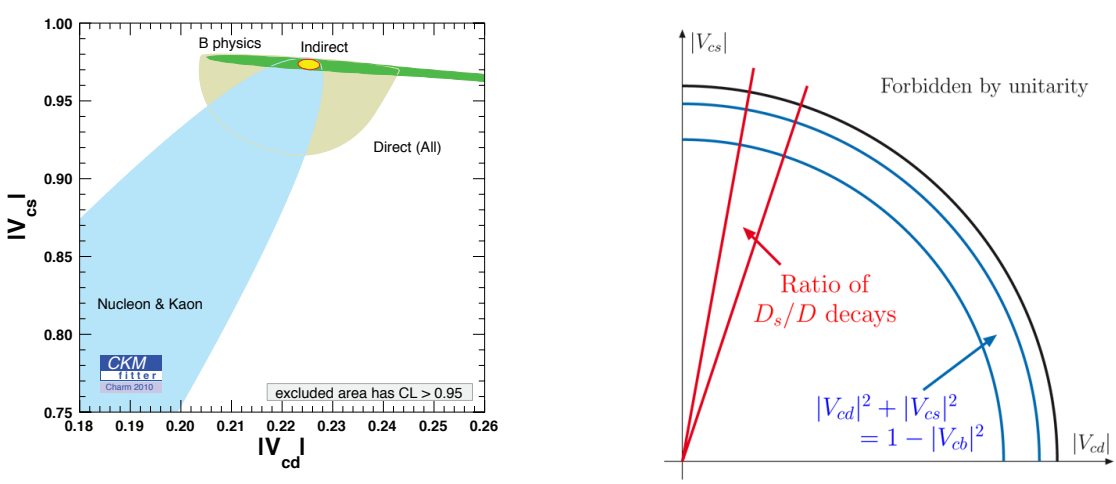

Fig. 3. On the left, constraints on $\left|V_{c d}\right|$ and $\left|V_{c s}\right|$ from the global fit, considering only inputs related to nucleon and kaon physics (in blue), $B$-physics (in green), and combined (in yellow), compared to the constraints from leptonic and semileptonic $D$-decays (in grey). On the right, a figure illustrating how ratios of $D$ and $D_{s}$ decays are complementary to indirect constraints from the measurements from $b \rightarrow c$ decays.

\section{Charm decays and physics beyond the Standard Model}

The global fit exhibits currently a discrepancy between the various constraints: its $\chi_{\min }^{2}$ drops by $\sim 2.4 \sigma[2.6 \sigma]$ if $\sin 2 \beta_{c \bar{c}}[B \rightarrow \tau \nu]$ is removed. One can invoke a fluctuation in the experimental measurements (not favoured by the good agreement between Babar and Belle) or in the theoretical inputs (requiring a correlated change in lattice estimates of $f_{B_{d}}$ and $B_{B_{d}}$ to change the prediction for $B \rightarrow \tau \nu$ without affecting $B_{d}$ mixing). One may also consider various extensions of the Standard Model to reproduce this situation, by introducing new Physics in $\Delta F=2$ processes $^{7}$ (and modifying $\sin (2 \beta)$ among others) or in $\Delta F=1$ processes (and altering $B \rightarrow \tau \nu$ and other quantities). As highlighted earlier, in the first case, $D$-mixing is difficult to exploit fully due to the issue of long-distance effects. In the latter case, $D_{(s)}$ decays may play a significant role to constrain new physics parameters. We focus on the example of the two-Higgs doublet model of type II $(2 \mathrm{HDMII})^{8}$. This model, where a Higgs doublet couples to down-type quarks and another one to up-type quarks (and to leptons), looks very much like the SM in the quark sector: the CKM matrix remains the only source of flavour changing interactions and there are no flavour-changing neutral currents at tree level. But there are new flavour-changing charged interactions, through the exchange of a charged Higgs instead a $W$ boson. Indeed, this model include several Higgs bosons (charged and neutral), and the two Higgs doublets take part in electroweak symmetry breaking, parametrised by the ratio of their vacuum expectation values $\tan \beta$.

Several decays are potentially sensitive to contributions from a charged Higgs, with a good control of the theoretical hadronic uncertainties: the leptonic decays of $K, \pi, D, D_{s}$ and $B$ mesons; the semileptonic decays $B \rightarrow D \ell \nu$ and $K \rightarrow \pi \ell \nu$; 

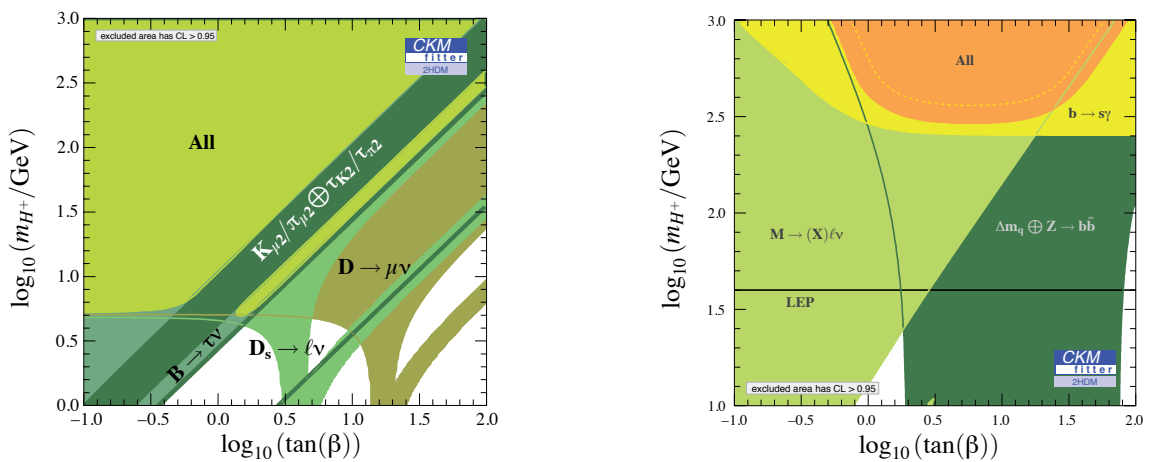

Fig. 4. Constraints on $(\tan \beta)$ and the mass of the charged Higgs boson in the two-Higgs doublet model of type II, considering the different leptonic decays (shades of green) and their combination (All - light green) [on the left] and all flavour observables [on the right].

the $Z$ partial width into $b$ quarks; the $\mathrm{FCNC}$ radiative decay $b \rightarrow s \gamma$. On the left hand-side of Fig. 4, we see how the overlap of leptonic decays, in particular $D$ and $D_{s}$ decays, helps in selecting allowed regions of the $\left(\tan \beta, M_{H^{+}}\right)$plane, corresponding either to the decoupling limit (with a very large $M_{H^{+}}$, leading to a tiny contribution to leptonic decays) or to a fine-tuned solution (corresponding to a particular value of the ratio $M_{H^{+}} / \tan \beta$ ) The right hand-side of Fig. 4 shows that the most stringent constraints come from leptonic decays and $b \rightarrow s \gamma$. The minimum $\chi^{2} \simeq 11$ is obtained for $m_{H^{+}}>600 \mathrm{GeV}$, with a discrepancy between the large measured $\mathcal{B}[B \rightarrow \tau \nu]$ favouring a fine-tuned solution at low $m_{H^{+}}$values, and all the other observables, in fair agreement with the SM expectations which select large values for the charged Higgs mass. We infer a lower limit of the charged Higgs mass $\left(m_{H^{+}}>323 \mathrm{GeV}\right.$ at $\left.95 \% \mathrm{CL}\right)$, but no significant constraint for $\tan \beta$.

\section{Acknowledgments}

I thank my colleagues of the CKMfitter group for a very enjoyable collaboration, and Hai-bo Li for his kind invitation. Work partially supported by PICS France-China.

\section{References}

1. The CKMfitter Group (J. Charles et al.), Eur. Phys. J. C41, 1-131 (2005), updated results and plots available at: http://ckmfitter.in2p3.fr

2. K. Trabelsi, Nucl. Phys. Proc. Suppl. 170 (2007) 70.

3. Talks and proceedings by J. Libby, D. Asner, J. Charles at this conference.

4. J. Charles et al., Phys. Rev. D 81 (2010) 054032 [arXiv:0912.0899 [hep-ph]].

5. Talk and proceedings by A. Lenz at this conference.

6. Talks and proceedings by H. Na, R. Poling, J. Simone and R. Briere at this conference.

7. A. Lenz et al., arXiv:1008.1593 [hep-ph].

8. O. Deschamps et al., Phys. Rev. D 82 (2010) 073012 [arXiv:0907.5135 [hep-ph]]. 